\title{
Developmental stage-specific effects of Pim-1 dysregulation on murine bone marrow B cell development
}

\author{
Zhihui Xu ${ }^{1,2}$, Kimberly A. Gwin'2, Yulin Li $i^{1,3^{*}}$ and Kay L. Medina ${ }^{2^{*}}$
}

\begin{abstract}
Background: The serine threonine kinase Pim-1 has documented roles in hematopoietic progenitor and B cell precursor proliferation and survival. Pim-1 is a molecular target of the transcription factor Hoxa9. Previous studies showed that Pim-1 deficiency phenocopied the hematopoietic progenitor defect in hoxa9-/- mice and forced expression of Pim-1 normalized the in vitro proliferation defect inherent to hoxa9-/- hematopoietic progenitors. Pim-1 is induced by cytokine signaling, including the early lymphoid/B lineage regulators Flt3 and IL-7, and expression levels were shown to influence the size of the B cell compartment in bone marrow (BM).

Results: In this study, we sought to determine if transgenic expression of Pim-1, driven by the

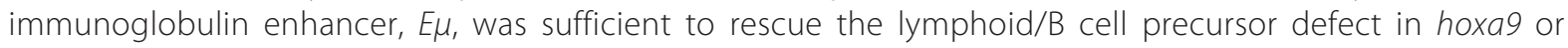
flt3-ligand (flt3l) deficient mice. Unexpectedly, expression of E $\mu$ - Pim1 exacerbated lymphoid progenitor deficiencies in flt3/-/-, and to a lesser extent, hoxa9-/- mice. Furthermore, E $\mu$-Pim 1 expression alone reduced early myeloid and lymphoid, but not erythroid, progenitors. In contrast, Pim-1 deficiency had no significant effect on early lymphoid/B cell development through the Pre-Pro-B cell stage, but caused a significant reduction in IgM $^{-}$B cell precursors. Importantly, loss of Pim-1 did not phenocopy hoxa9- or flt3l-deficiency on the lymphoid/early B cell progenitor pools.
\end{abstract}

Conclusions: These experimental findings demonstrate that Pim-1 overexpression has developmental-stage-specific effects on B lymphopoiesis and myelopoiesis. Importantly, these suggest that Pim-1 deficiency does not contribute significantly to the early lymphoid/B cell developmental deficiency in hoxa9-/- or flt3/-/- mice.

Keywords: B cell precursors, EM-Pim1 transgene, pim1-/-, Flt3, Hoxa9, B cell development, Lymphopoiesis, Hematopoiesis

\section{Background}

The steady-state production of B lineage lymphocytes in bone marrow is contingent on the developmental-stagespecific expression and combinatorial activities of multiple regulatory proteins including transcription factors, signaling molecules, microRNAs, and epigenetic modifiers. A critical early event in B cell development is lymphoid

\footnotetext{
* Correspondence: ylli@mail.ju.edu.cn; medina.kay@mayo.edu

${ }^{1}$ The Key Laboratory Pathobiology, Ministry of Education, Norman Bethune College of Medicine, Jilin University, Changchun 130000, People's Republic of China

${ }^{2}$ Department of Immunology, College of Medicine, Mayo Clinic, 200 First Street SW, Rochester, MN 55905, USA

Full list of author information is available at the end of the article
}

lineage specification within the multipotential progenitor (MPP) pool which we and others have shown is dependent, in part, on the homeodomain transcription factor Hoxa9 and signaling via the receptor tyrosine kinase Flt3 [1-4]. Germline knockout of Hoxa9 or Flt3-ligand largely phenocopy each other with respect to reductions in numbers of MPPs, the lymphoid progenitor subsets ALP and BLP, and B cell precursors in BM. Importantly, Hoxa9 and Flt3 signaling function synergistically to regulate a critical early checkpoint in lymphoid development, as combined loss of Hoxa9 and Flt3 signaling ablates the generation of lymphoid primed multipotential progenitors [4]. 
Pim-1 is a member of the Pim family of protooncogenes that encode a distinct class of serine/threonine kinases. Pim-1 was originally identified as a target for proviral activation in Moloney murine leukemia virus induced $\mathrm{T}$ cell lymphomas [5]. PIM proteins are widely expressed in most tissues and co-expression of family members has been found in various tissues [6]. The transcription of Pim family members is induced by a variety of cytokines and mitogens that transduce their primary signal through the JAK/STAT pathway [7]. A role for Pim-1 in B cell development was previously demonstrated through analysis of pim1-/- and E $\mu$-Pim1 transgenic mice $[8,9]$. Pim1-/- Pre-B cells exhibited impaired in vitro proliferation in response to IL-7 and stem cell factor (SCF) that was rescued by expression of a functional Pim-1 transgene [10]. In contrast, overexpression of Pim-1increased numbers of IL-7 + SCF responsive $\mathrm{B}$ cell colonies. These combined data provided the first evidence that Pim-1 was an important regulator of B lymphopoiesis in mice, and linked Pim-1 to the IL-7R signaling pathway.

Cytokine signaling plays an essential role in early lymphoid/B cell development. Threshold levels of Flt3 signaling are required for the proliferation, survival, and maintenance of MPPs competent to generate B cell precursors $[1,11]$. Flt3 signaling is mediated by the Ras and STAT5 pathways [12]. A dominant negative form of Ras was shown to phenocopy the B lineage developmental block in flt3-/- mice, impairing the proliferation of common lymphoid progenitors and Pre-Pro-B cells. The same study showed that Ras promoted STAT5-dependent Pro-B differentiation by enhancing expression of IL-7R $\alpha$ [12]. Pim-1 is induced downstream of Jak2/STAT5 signaling and has also been implicated in playing a role in the proliferation and/or differentiation of myeloid progenitors [13-15]. Importantly, a role for Pim-1 in regulation of the early lymphoid/B cell progenitor pool, prior to expression of CD45R/B220, has not been reported.

Functional studies have confirmed a role for Pim-1 in regulating hematopoietic stem cell (HSC) proliferation and survival. HSCs from pim1-/- mice exhibited impaired repopulating capacity in competitive transplantation experiments [16]. In vitro assays revealed decreased cytokine mediated cell growth and differentiation of hematopoietic progenitors [7]. In contrast, overexpression of human Pim-1 driven by vav hematopoietic regulatory elements and SV40 showed enhanced hematopoietic progenitor function in vitro and in vivo [16]. The hematopoietic defects exhibited by pim1-/mice are strikingly similar to those in hoxa9-/- mice as loss of hoxa9 also impaired the proliferation and repopulating ability of HSCs [17]. Consistent with this observation, pim1 is a direct target of Hoxa9 [18]. Somatic ablation of pim1, hoxa9, or flt3 causes select reductions in hematopoietic progenitor subsets and $\mathrm{B}$ cell precursors. However, an obligate role for Pim-1 in regulation of lymphoid and/or early B cell development has not been investigated. In this study we evaluated the role of Pim-1 in murine lymphoid lineage specification and $B$ cell development through comparative flow cytometric analysis of hoxa9-/- $x$ E

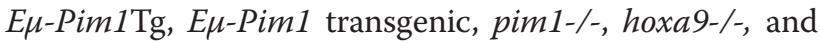
flt3l-/- mice. Our experimental findings revealed that Pim-1 dysregulation has developmental-stage-specific effects on B lymphopoiesis and early myeloid, but not erythroid progenitors. Furthermore, we show that Pim1deficiency is not the basis of the lymphoid or early B cell developmental defects in hoxa9-/- or flt $3 \mathrm{l} /$ /- mice.

\section{Methods \\ Mice}

Wildtype C57Bl/6 mice were generated from our breeding colony. Pim1-/- and E $\mu$ - Pim1 transgenic mice have been previously described [10]. Pim1-/- mice were provided by Andrew S. Kraft and E $\mu$ - Pim1 transgenic mice were provided by Jung-Hyun Park and permission for both obtained from A. Berns. All mice evaluated in this

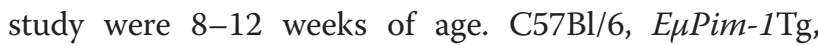
hoxa9-/- $x$ E were bred in the Mayo Clinic animal facility, and all animal experiments detailed in this study were conducted under guidelines established and approved by the Mayo Clinic Institutional Animal Care and Use Committee. PCR, using DNA isolated from tail clips, was performed using previously published primers to confirm the genotype of compound mice $[19,20]$.

\section{Flow cytometric analysis of BM hematopoietic progenitor} subsets and $B$ cell precursors

Methods for flow cytometry and progenitor isolation have been described [4]. Flow cytometric analysis was performed on the FACS-Canto or LSRII cytometers (BD BioSciences, San Jose, CA) and data analysis performed with FlowJo software (Tree Star, Ashland, OR). All antibodies used in this study were purchased from eBioScience or Biolegend. HSC/MPPs were resolved using the following combinations of antibodies: Lineage positive APC cocktail (CD3e, Ter119, B220, Mac-1, Gr1), CD34 FITC, Flt3 PE, Sca-1 PerCP-Cy5.5, CD150 PECy7, c-kit APC-eflour 780 and $1.5 \times 10^{6}$ million events collected for the analysis. MEPs, CMPs, and GMPs were resolved using the following combinations of antibodies: Lineage positive APC cocktail (CD3e, Ter119, B220, Mac-1, Gr-1), CD34 FITC, Flt3 PE, Sca-1 PerCP-Cy5.5, c-kit APC-eflour 780 and $1.5 \times 10^{6}$ million events collected for the analysis. ALP and BLP were resolved using the following combinations of antibodies: Lineage positive FITC cocktail (CD3e, CD8 $\alpha$, CD11c, NK1.1, 
Ly6C, Ter119, B220, Mac-1, Gr-1), Flt3 PE, IL-7R PECy7, Ly6D AF647, c-kit APC-eflour 780, PDCA1 PerCPeflour 710 and $2 \times 10^{6}$ million events collected for the analysis. Pre-Pro-B and BCP subsets were resolved using the following combinations of antibodies: B220 FITC, AA4.1 PE, PDCA1 PerCP-eflour 710, CD19 Pe-Cy7, Ly6D AF647, and IgM APC-Cy7 and 500,000 to $1 \times 10^{6}$ million events collected for the analysis. Red blood cells and cellular debris were excluded from the analysis with mononuclear cell and doublet exclusion gates. Absolute cell numbers were determined by multiplying population frequencies (total subset event count divided by total event count) times mononuclear cell count. Cell counts reflect numbers of cells per 4 hindlimb leg bones.

\section{Realtime RT-PCR analysis of pim 1 transcript levels in bone marrow progenitor subsets}

Hematopoietic progenitor subsets were purified by cell sorting for RNA isolation, cDNA synthesis, and qPCR analysis as we previously described [21]. HSC/MPP were purified as $\mathrm{Lin}^{-}$(see $\mathrm{Lin}^{+}$cocktail above) c-kit ${ }^{\text {hi }} \mathrm{Sca}-1^{+}$ Flt3 $^{\text {-lo }}$, LMPP as Lin $^{-}$c-kit ${ }^{\text {hi }} \mathrm{Sca}^{+}{ }^{+} \mathrm{Flt}^{\text {hi }}{ }^{\text {, CLP }}$ as $\mathrm{Lin}^{-}$

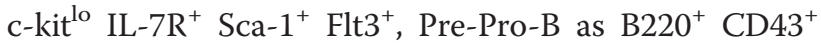
$\mathrm{CD}^{-} 9^{-} \mathrm{IgM}^{-}$(which includes a mix of Pre-Pro-B, NK, and pDCs), Pro-B as $\mathrm{B} 220^{+} \mathrm{CD}_{4} 3^{+} \mathrm{CD} 19^{+} \mathrm{IgM}^{-}$, Pre-B as $\mathrm{B}_{22}{ }^{+\mathrm{lo}} \mathrm{CD}_{4} 3^{-} \mathrm{CD}_{19}{ }^{+} \mathrm{IgM}^{-}$, and $\mathrm{IgM}^{+}$as $\mathrm{B} 220^{+\mathrm{hi}}$ $\mathrm{CD}_{4} 3^{-} \mathrm{CD}_{19}{ }^{+} \mathrm{IgM}^{+}$. Realtime PCR was performed using a pim1 taqman probe (Mm00435712_m1) and gene expression normalized to $18 \mathrm{~S}$ RNA. All cDNA samples were assayed in triplicate. Relative transcript abundance was determined using the $2^{-\Delta \Delta C T}$ method.

\section{Statistics}

Statistical significance was determined using the Student- $t$ test. Data are reported as standard error of the mean (SEM) and $p$-values less than 0.05 were considered significant.

\section{Results}

Forced expression of Pim-1 does not rescue the lymphoid/B lineage deficiency in hoxa9-/- or flt3l-/- mice

Gene-targeted ablation of pim1, hoxa9, or flt 3 causes reductions in select hematopoietic progenitor subsets and B cell precursors. Pim-1 is a molecular target of Hoxa9 and has been shown to be induced by Flt3 signaling $[18,22]$. To determine if dysregulated expression of Pim-1 contributed to the lymphoid/B lineage precursor deficiency in hoxa9-/- or flt3l-/- mice, hoxa9-/- $x$ E $\mu$ Pim1Tg and flt3l-/- $x$ E $\mu$-Pim1Tg mice were generated. Transgenic expression of E $\mu$-Pim1 had no significant effect on BM cellularity in hoxa9-/- or flt3l-/- mice (data not shown). Hoxa9-/- and flt3l-/- mice have significantly decreased numbers of Lin $^{-}$c-kit ${ }^{\text {lo }} \mathrm{IL}_{-} 7 \mathrm{R}^{+}$common lymphoid progenitors (CLPs) [4]. CLPs can be fractionated into
Lin $^{-}$PDCA1 $^{-}$IL-7R $^{+}$c-kit ${ }^{\text {lo }}$ Flt3 $^{+}$Ly6D $^{-}$all lymphoid progenitors (ALP) and $\mathrm{Lin}^{-} \mathrm{PDCA}^{-} \mathrm{IL}^{-7 \mathrm{R}^{+}} \mathrm{c}^{-k i t^{\mathrm{lo}}} \mathrm{Flt3}^{+}$

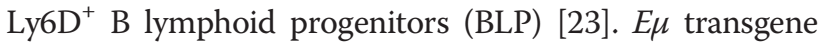
driven expression of Pim-1 did not exacerbate the deficiencies in ALP or BLP in hoxa9-/- mice (Fig. 1a-c). However, E $\mu$-Pim1Tg expression did exacerbate the reductions in ALP and BLP in flt3l-/- mice (Fig. 1a-c). Expression of B220 distinguishes Pre-Pro-B cells from BLP [23]. Consistent with reductions in ALP and BLP, numbers of $\mathrm{PDCA}^{-}$Ly6D $^{+} \mathrm{B}^{2} 20^{+} \mathrm{CD}^{-} 9^{-}$Pre-Pro-B cells were

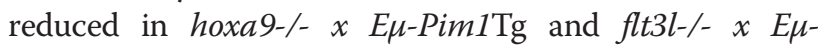
Pim1Tg mice compared to WT (Fig. 2a-b). However, in contrast to ALP and BLP, E $\mu$-Pim1Tg expression did not abrogate the Pre-Pro-B cell deficiency hoxa9-/- or flt3l-/mice (Fig. 2a-b). The B lineage committed progeny of Pre-Pro-B cells are $\mathrm{IgM}^{-} \mathrm{B}_{220^{+}} \mathrm{CD} 19^{+} \mathrm{B}$ cell precursors (BCPs). Unlike the negative impact of E $\mu$-Pim1 transgene expression on CLP subsets and Pre-Pro-B cells, no statistically significant reduction in $\mathrm{IgM}^{-} \mathrm{B} 220^{+} \mathrm{CD} 19^{+} \mathrm{BCPs}$ or

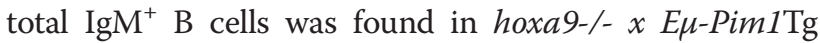
(Fig. 2a,c-d). However, reductions in $\mathrm{IgM}^{-} \mathrm{B} 220^{+} \mathrm{CD}^{+} 9^{+}$ $\mathrm{BCP}$ and total $\operatorname{IgM}^{+} \mathrm{B}$ cell compartments were maintained in flt3l-/- and flt3l-/- $x$ E 4 -Pim1Tg mice (Fig. 2a,c-d). These data reveal a previously uncharacterized inhibitory effect of E $\mu$-Pim1Tg expression on early lymphoid/B cell development.

\section{Impact of Pim1-deficiency versus $E \mu$ - transgene driven expression of Pim-1 on BM lymphoid progenitors and BCPs}

A role for Pim-1 in B cell development was previously demonstrated from analysis of pim1-/- and E $\mu$-Pim1 transgenic mice [10]. Our findings that $E \mu$ transgene driven expression of Pim-1 abrogated lymphoid/early B cell development supported further evaluation of the effects of dysregulated expression of Pim-1 on development of this lineage. BM cells from WT, pim1-/-, and E $\mu$-Pim1 transgenic mice were stained with combinations of antibodies to resolve ALP, BLP, Pre-Pro-B, IgM ${ }^{-}$ $\mathrm{BCP}$, and $\operatorname{IgM}^{+} \mathrm{B}$ cells. Comparison of BM mononuclear cell counts revealed no significant difference between WT and E $\mu$-Pim1 transgenic mice, but a statistically significant reduction in pim1-/- mice $\left(5.2 \times 10^{7} \pm 0.33, n=3\right.$; $p=0.0055)$ compared to WT $\left(7.9 \times 10^{7} \pm 1.3, n=10\right)$. No statistically significant alterations in absolute numbers of ALP, BLP, or Pre-Pro-B were identified between WT and pim1-/- mice (Fig. 3a). The inverse was found in E Pim1 transgenic mice wherein absolute numbers of phenotypic ALP, BLP, and Pre-Pro-B cells were significantly reduced compared to WT (Fig. 3a). Finally, while numbers of $\operatorname{IgM}^{-} \mathrm{BCP}$ and $\operatorname{IgM}^{+} \mathrm{B}$ cells in E $\mu$-Pim1 transgenic mice were comparable to WT (Fig. 3a), we found that $\operatorname{IgM}^{-}$, but not $\operatorname{IgM}^{+}, B C P s$ were reduced in pim1-/- mice [10]. CD19 ${ }^{+}$IgM $^{-}$BCPs constitute Pro-B 


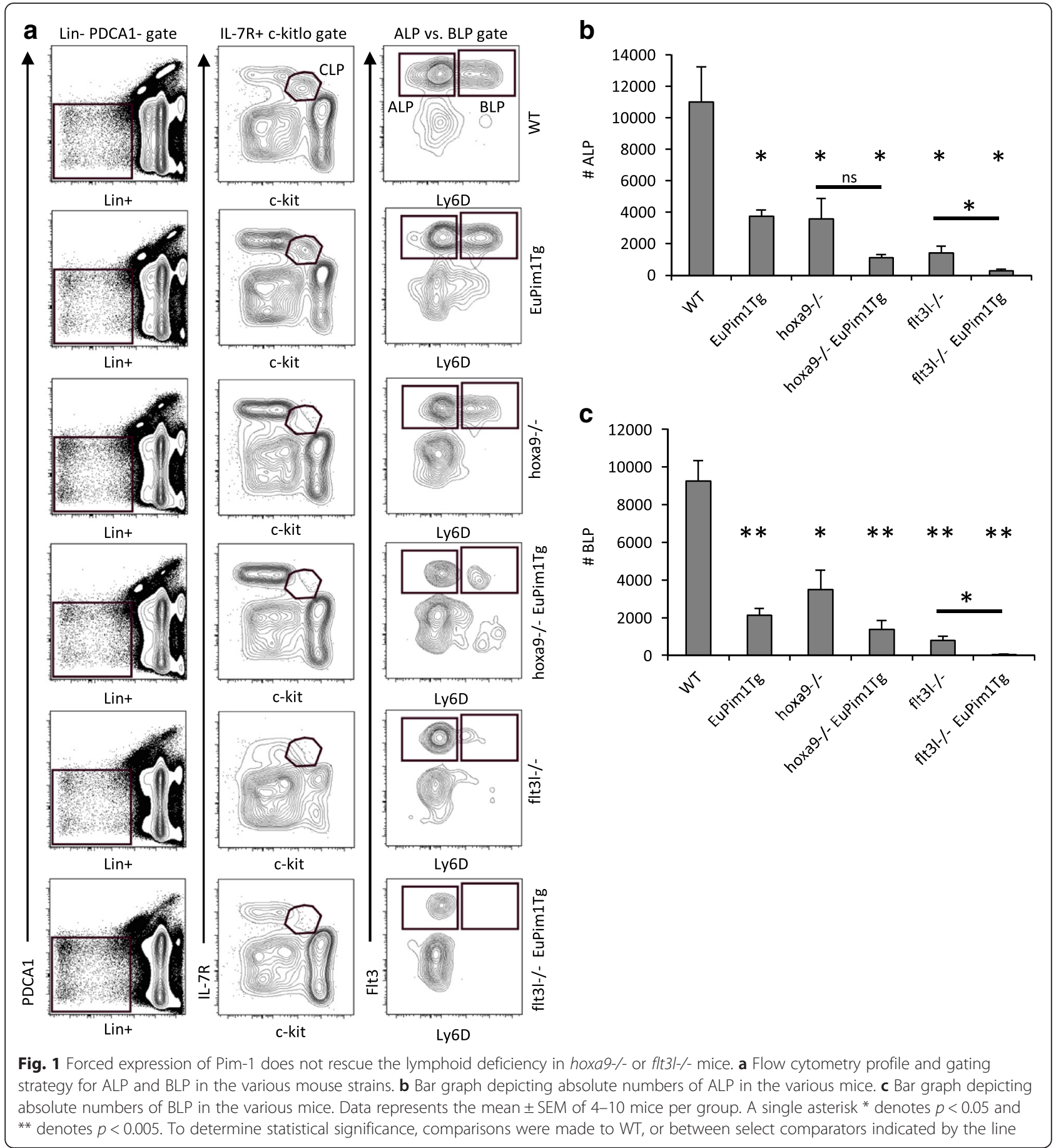

and Pre-B cells. To gain further insight into the role of Pim-1 in hematopoietic development and B cell differentiation, we evaluated pim-1 transcript levels in purified bone marrow progenitor subsets. As shown in Fig. 3b, abundant pim-1 transcript expression was high exclusively in Pre-B cells across the B lineage developmental spectrum. Taken together, these data suggest that elevated levels of Pim-1 are inhibitory for lymphoid/B cell development prior to commitment to the B cell fate.
On the other hand, loss of Pim- 1 decreased IgM $^{-}$Pro-B/ Pre-B cells, suggesting an essential role at this stage.

Impact of Pim1-deficiency versus $E \mu$ - transgene driven expression of Pim-1 on HSC, MPP, and erythroid/myeloid progenitors

The $E \mu$ enhancer is active very early in hematopoiesis, within $\mathrm{LSK}^{+}$MPPs [11, 24]. A previous study reported increased numbers of $\mathrm{LSK}^{+}$cells in vav-Pim1Tg mice, 


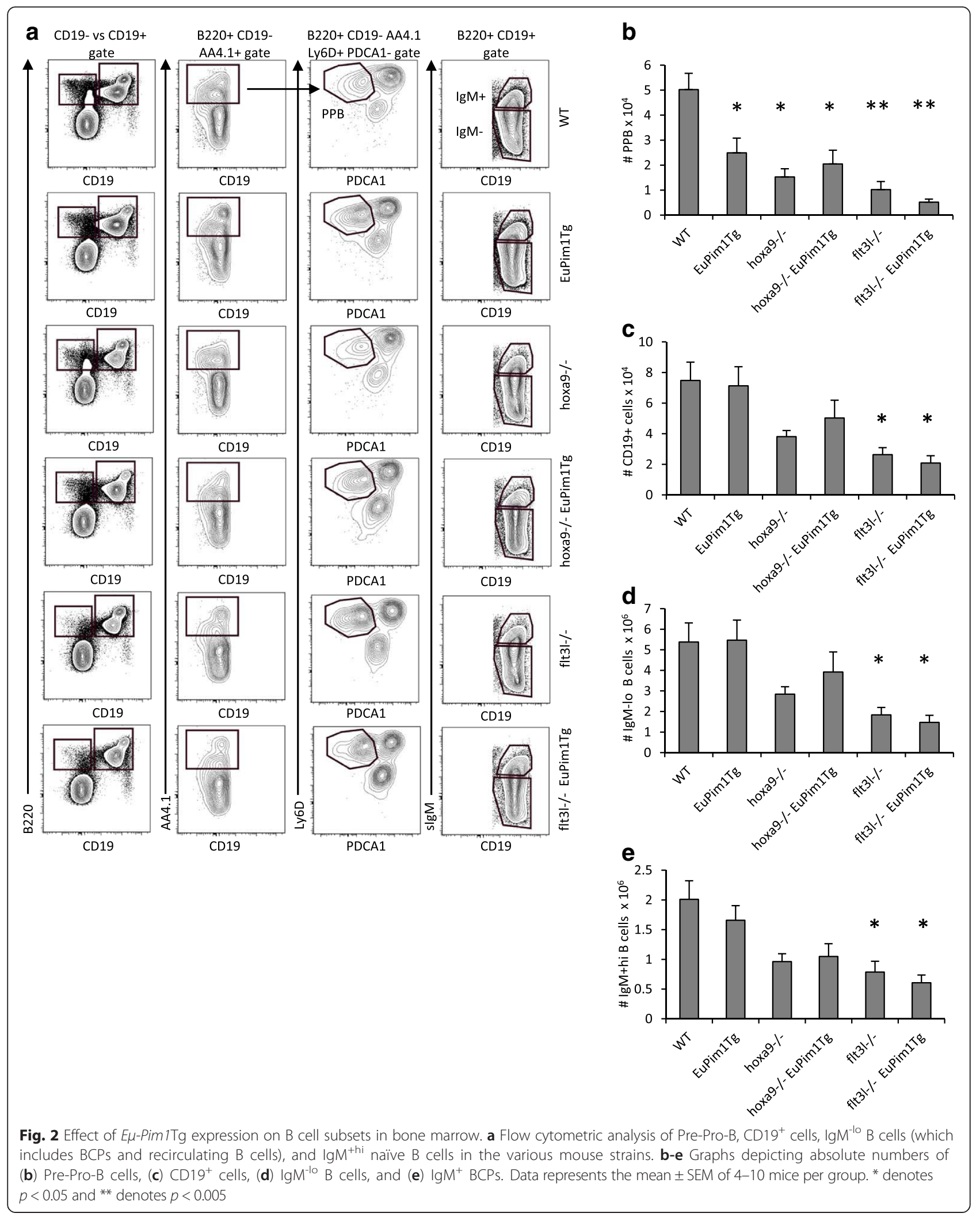




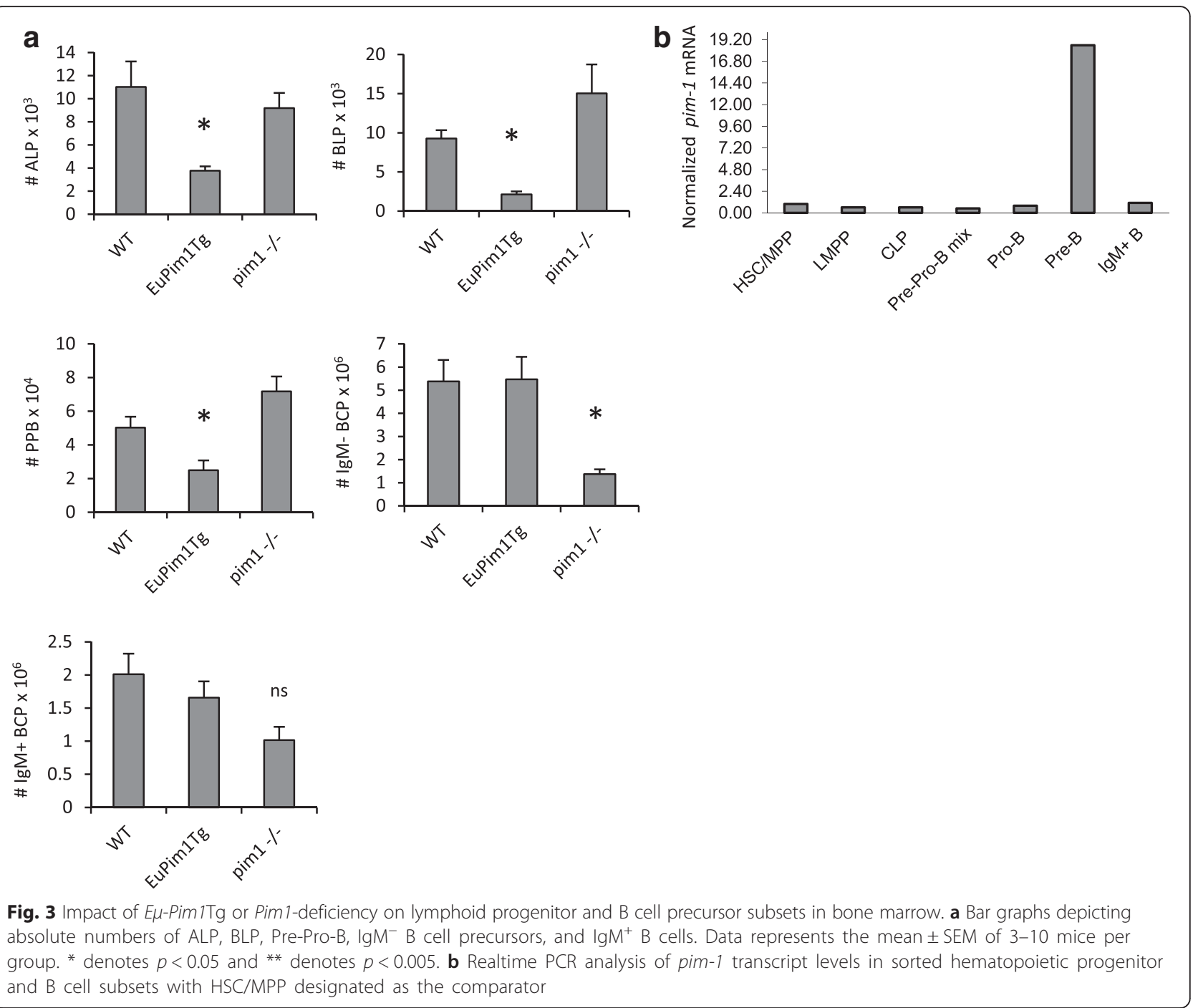

but a similar analysis has not been reported for $E \mu$ Pim1Tg mice [16]. To begin, we compared frequencies of $\mathrm{LSK}^{+}$cells. In contrast to results reported for vavPim1Tg mice, frequencies and numbers of total $\mathrm{LSK}^{+}$ cells were significantly reduced in E $\mu$-Pim $1 \mathrm{Tg}$ mice (Fig. 4a-b and data not shown). Next we compared frequencies and numbers of $\mathrm{LSK}^{+} \mathrm{CD} 150^{+} \mathrm{CD} 34^{-} \mathrm{HSC}$ and $\mathrm{LSK}^{+} \mathrm{CD}_{150^{-}} \mathrm{Flt3}^{+} \mathrm{MPP}$ in E$\mu$-Pim1Tg mice. Absolute numbers of HSC and MPP were significantly reduced in E $\mathrm{A}$-Pim1Tg mice (Fig. 4c-d). However, the reduction was attributed to the total decrease in $\mathrm{LSK}^{+}$ cells as percentages of $\mathrm{CD} 150^{+} \mathrm{CD} 34^{-}$HSC within the $\mathrm{LSK}^{+}$compartment were increased in E $\mu$-Pim1Tg mice $(6.23 \pm 0.990$ vs. $4.05 \pm 1.07 \%, p=0.014$, in E $\mu$-Pim $1 \mathrm{Tg}$ vs. WT, respectively). The same was true for the reduction in absolute numbers of MPP as percentages $\mathrm{CD}_{150}{ }^{-} \mathrm{Flt3}^{+}$within the $\mathrm{LSK}^{+}$compartment was not significantly reduced $(73.7 \pm 4.53$ vs. $80.1 \pm 3.72 \%, p=0.064$, in $E \mu$-Pim $1 \mathrm{Tg}$ vs. WT, respectively). The reduction in

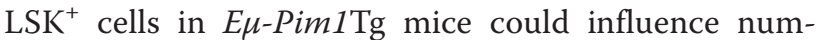
bers of erythroid/myeloid progenitors. Frequencies of $\mathrm{Lin}^{-} \mathrm{ckit}^{\text {hi }} \mathrm{Sca}^{-}$cells were not altered in E $\mu$-Pim1Tg mice (Fig. 4a). However, significant alterations in frequencies and absolute numbers of CMP and GMP, but not MEP, were documented E $\mu$-Pim1Tg mice (Fig. 4a,e-m). No significant alterations in frequencies or numbers of $\mathrm{LSK}^{+}, \mathrm{HSC}$, MPP, CMP, MEP, or GMP were observed in pim1-/- mice, compared to WT (Fig. 4). Taken together, these data show that E $\mu$-Pim1Tg driven expression of Pim-1, but not Pim1-deficiency, reduces the myeloid but not the erythroid/megakaryocytic progenitor pools.

Loss of Pim-1 does not phenocopy hoxa9- deficiency on the lymphoid/early B cell progenitor pools

Pim-1 is a Hoxa9 target gene and forced expression of Pim-1 rescued the in vitro proliferation of hoxa9-/- BM progenitors [18]. We reasoned that if Pim1-deficiency is 

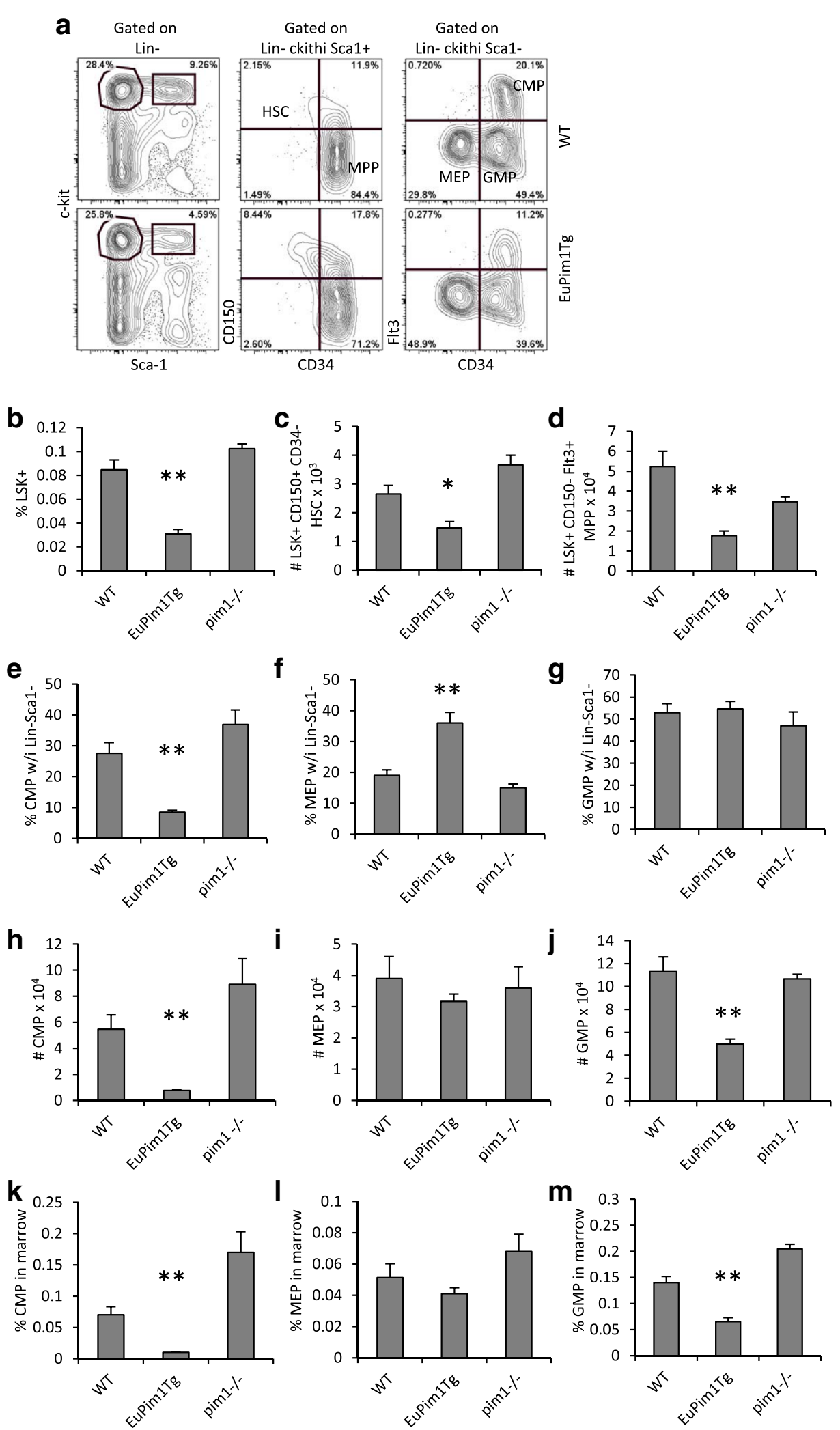

Fig. 4 (See legend on next page.) 
(See figure on previous page.)

Fig. 4 Impact of E $\mu$-Pim 1Tg or Pim1-deficiency on primitive hematopoietic and myeloid-erythroid progenitor subsets in bone marrow. a Flow cytometry profile and gating strategy for HSC, Flt3+ MPP, CMP, GMP, and MEP. Bar graphs depict absolute numbers or frequencies of the various progenitor subsets. b Percentage of LSK+ in total BM. c-d Absolute number of HSC or MPP in BM. e-g Frequencies of CMP, MEP, or GMP within the Lin- ckithi Sca1- subset. $\mathbf{h}-\mathbf{j}$ Absolute numbers of CMP, MEP, or GMP in BM. $\mathbf{k}-\mathbf{m}$ Frequencies of CMP, MEP, or GMP in BM. Data represents the mean \pm SEM of 3-10 mice per group. * denotes $p<0.05$ and ${ }^{* *}$ denotes $p<0.005$

the basis of the hematopoietic progenitor defect in hoxa9-/- mice, then pim1-/- mice should phenocopy, at least in part, hoxa9-/- progenitor defects in BM. Hoxa9-/- and pim1-/- mice shared statistically significantly increased frequencies of HSCs. However, while hoxa9-/- and flt3l-/- mice shared similar reductions in CMP, GMP, ALP, BLP, and Pre-Pro-B cells, consistent with our previous observation, loss of Pim-1 did not phenocopy Hoxa9-deficiency (Fig. 5) [4, 23].

\section{Discussion}

Previous studies have implicated a role for Pim-1 in regulation of $\mathrm{B}$ cell development in BM and pim-1 is a Hoxa9 target gene [10, 18]. Furthermore, hoxa9- and pim1-deficient mice share similar hematopoietic phenotypes and forced expression of Pim-1 rescued the in vitro proliferation defect in hoxa9-/- BM progenitors $[17,18]$. To determine if forced expression of Pim-1 was sufficient to restore the lymphoid progenitor/B cell precursor defect in hoxa9-/- or flt3l-/- mice, we gener-

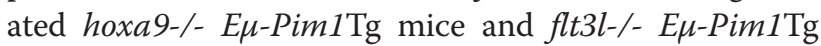
mice. Unexpectedly, $E \mu$ driven expression of Pim-1 exacerbated the deficiencies in ALP and BLP in flt3l-/-, but not hoxa9-/- mice. The detrimental effect in $f l t 3 l-/-$ $E \mu$-Pim1Tg mice was specific to the ALP and BLP stages of $\mathrm{B}$ cell differentiation, as the E $\mu$-Pim1Tg did not further exacerbate the B cell deficiencies in $\mathrm{flt} 3 \mathrm{l} / \mathrm{-}-\mathrm{-mice}$ from the Pre-Pro-B through the $\operatorname{IgM}^{+}$stages. The negative effect of $E \mu$-Pim1Tg expression was not restricted to the ALP and BLP compartments. We previously showed that $E \mu$ is active from a very early stage in hematopoiesis and here we also show that E $\mu$-Pim1Tg expression caused significant reductions in numbers of HSCs, MPPs, CMPs, and GMPs suggesting that Pim-1 expression levels must be carefully controlled during early hematopoietic differentiation [11, 24]. In contrast, loss of Pim-1, which we hypothesized might have deleterious consequences on the early lymphoid/B cell progenitor pools, was dispensable for hematopoiesis with the exception of IgM $^{-}$BCPs [10]. Consistent, with our findings, realtime PCR of pim-1 transcription across the hematopoietic spectrum, showed significant levels limited to BCPs at the Pre-B cell stage. Finally, through direct comparative hematopoietic progenitor subset analysis of hoxa9-/-,
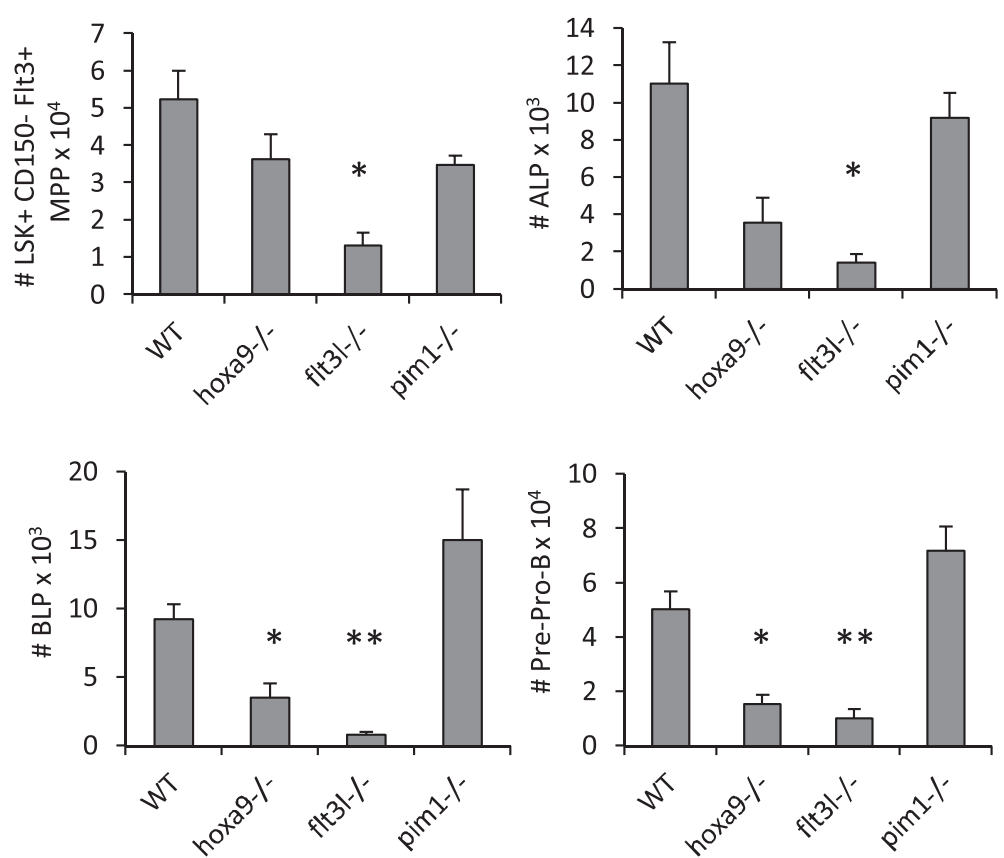

Fig. 5 Comparison of Hoxa9-, F/3tl-, and Pim1-deficiencies on hematopoietic progenitor subsets and Pre-Pro-B cells. Bar graphs depict absolute numbers of the various progenitor subsets. Data represents the mean \pm SEM of 3-4 mice per single knockout and 10 WT mice. ${ }^{*}$ denotes $p<0.05$ and ${ }^{* *}$ denotes $p<0.005$ 
flt3l-/-, and pim1-/- mice, we suggest that loss of Pim-1 is unlikely to contribute significantly to the hematopoietic progenitor defects in either hoxa9-/- or flt3l-/- mice. Taken together, these comprehensive flow cytometric analyses demonstrate very select lineage and developmentalstage-specific consequences of dysregulated expression of Pim-1 expression on BM hematopoietic progenitor and B lineage subsets.

The serine threonine phosphokinase Pim-1 is highly expressed in hematopoietic cells in mouse and man $[20,25]$. The pim-1 gene was originally discovered as a common insertion site in MoMuLV-induced $\mathrm{T}$ cell lymphomas and later found in B-cell lymphomas and erythroleukemias. Overexpression of Pim-1 driven by the $E \mu$ enhancer was shown to lead to a low incidence of $\mathrm{T}$ cell lymphomas and increased sensitivity to chemically induced $\mathrm{T}$ cell transformation. In the $\mathrm{B}$ lineage, overexpression of $E \mu$ - Pim-1 facilitated establishment of B cell progenitor cell lines blocked at the Pre-B cell stage of differentiation [10]. The same study reported that marrows with the highest Pim-1 expression had a reduction in M-CSF responsive cells, and suggested the result could be due to impaired macrophage differentiation or on a macrophage pro-

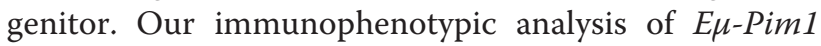
transgenic mice provides insight into the reductions in M-CSF responsive cells as we identified significant reductions in frequencies and absolute numbers of CMP and GMP.

PIM kinases are short-lived proteins induced at the level of transcription upon mitogenic stimulation [26]. They do not have regulatory domains and are constitutively active when expressed. Under physiologic conditions, their short half-life limits their activity [27]. Many cytokines that induce pim gene transcription act through the JAK/STAT pathway. The pim1 promoter has binding sites for STAT3 and STAT5 [28, 29]. Pim-1 protein then, in turn, functions to inhibit the JAK/STAT pathway by binding and activating SOCS proteins [30]. SOCS proteins support cytokine signaling through the Ras/MAPK pathway by binding the Ras inhibitor RasGAP [30]. Interestingly, dysregulated Ras/SOCS function mediated by overexpression of Pim-1 provides a plausible cellular mechanism for the impaired lymphoid/early B lineage development exhibited by $E \mu$ - Pim $1 \mathrm{Tg}$ mice. Ras proteins are involved in regulating proliferation and differentiation of various cell types in a tissue specific manner. Pertinent to this study, mice expressing a dominant negative form of human H-Ras have a severe block in B cell development at the Pre-Pro-B to Pro-B transition [31]. Li, et al., previously showed that a Flt3/Ras dependent signal governs lymphoid progenitor (ALP + BLP) and Pre-Pro-B proliferation [12]. In addition, they showed that Flt3/Ras suppresses expression of socs 2 and socs 3 . They concluded that Flt3 activation of Ras primes B cell development by inducing a state of STAT5 responsiveness, a key event downstream of IL-7R signaling in lymphoid progenitors leading to induction of the $\mathrm{B}$ cell fate specification and commitment factor EBF1. Sustained expression of Pim-1 would be predicted to stabilize SOCS expression, impairing STAT5-mediated induction of ebf1, suppressing EBF1directed B cell differentiation. Indeed, this hypothetical molecular explanation is consistent with our findings that the Flt3 dependent ALP and BLP stages are exquisitely sensitive to dysregulated Pim-1 in flt3l-/- mice. It also suggests that it is unlikely that elevated levels of Pim-1 are inhibitory for lymphoid/B cell development by accelerating B cell differentiation, given the critical role for STAT5/ EBF1 in this process.

Pim kinases act as oncogenes by promoting cell cycle progression by phosphorylating and down-regulating the cyclin dependent kinase inhibitor p27Kip1. Pim kinases also have the ability to suppress p27kip1 transcription through phosphorylation and inactivation of forkhead transcription factors, including FoxO1 and FoxO3 [32]. Pim-1 inactivation of FoxO1 would have deleterious effects on B cell fate determination as FoxO1 is an integral member of a global network of transcription factors, including E2A and EBF1 [33]. Importantly, FoxO1-deficient mice also have an arrest in B cell development at the CLP stage, specifically at the BLP stage, and phenocopy EBF1-deficiency [34]. Importantly, FoxO1-deficient BLPs have increased transcripts for socs2, reminiscent of Pim-1 overexpression [30]. Based on our immunophenotyping results, together with previous phenotypic, molecular, and cellular data in the literature database, we hypothesize that dyregulated expression on Pim-1, driven by $E \mu$, impairs lymphoid/early B cell differentiation through upregulation of SOCS proteins and inactivation of FoxO1. Upregulation of SOCS proteins inhibits STAT5 mediated B cell differentiation leading to upregulation of EBF1. Inactivation of FoxO1 impairs the global transcriptional network orchestrated by E2A, EBF1, and FoxO1 that directs the early program of $\mathrm{B}$ lineage gene expression, requisite for $B$ cell differentiation. In contrast, loss of Pim-1, would be predicted to suppress SOCS protein expression and stabilize FoxO1, favoring B cell developmental potential, providing an explanation for the slight, but not statistically significant, increase in BLP and Pre-Pro-B cells we observed in pim1-/- mice. We note that additional studies are required to determine if this molecular explanation is valid.

The goal of this project was to investigate the role of Pim-1 in early lymphoid/B cell development. However, we also found reductions in numbers of HSC, MPP, CMP, and GMP in E $\mu$-Pim1Tg mice. An, et al., reported an increase in frequencies and numbers of $\mathrm{LSK}^{+}$ cells in vav-Pim1 Tg mice, whereas we report decreased 
frequencies and numbers of $\mathrm{LSK}^{+}$cells in $E \mu-P i m 1 \mathrm{Tg}$ mice [16]. The overall decrease in $\mathrm{LSK}^{+}$cells was the basis of the decreased numbers of HSCs and MPPs, as frequencies of $\mathrm{CD} 150^{+} \mathrm{CD} 34^{-}$immunophenotypic HSC within the $\mathrm{LSK}^{+}$compartment were actually increased in $E \mu$-Pim1Tg mice. We believe it unlikely that $E \mu-P i m 1 \mathrm{Tg}$ expression has any functional consequence on HSC biology for two reasons. First, we previously showed that $E \mu$ driven reporter expression is confined to $\mathrm{CD} 27^{+}$MPPs within the $\mathrm{LSK}^{+}$compartment and HSCs are CD27 $7^{-}[11,24,35]$. Second, the frequencies of $\mathrm{CD} 150^{+} \mathrm{CD} 34^{-}$HSCs were substantially increased in E $\mu$ - Pim1Tg mice.

The cellular and/or molecular basis of the inhibitory consequence of $\mathrm{E \mu}$-Pim1Tg expression on numbers of Flt3 ${ }^{+}$ MPPs in noteworthy, as Flt3 $3^{+}$MPPs are the precursors of CMP and CLP and these hematopoietic subsets were the most sensitive to $E \mu-P i m 1 \mathrm{Tg}$ expression. E $\mu-P i m 1 \mathrm{Tg}$ expression did not impact $\mathrm{Flt}^{+} \mathrm{CMP}$ or CLP by altering the expression levels of Flt3. However, Flt $3^{+}$CMP and CLP were particularly sensitive to $E \mu-P i m 1 \mathrm{Tg}$ expression. These findings suggest that overexpression of Pim-1 might negatively impact signaling networks downstream of Flt3 stimulation in $\mathrm{Flt}^{+}$progenitor subsets, impairing either their proliferation or survival. Regardless of the mechanism, differentiating CMP and CLP overcome the inhibitory effects and their downstream progeny are refractory to sustained expression of Pim-1. Overexpression of Pim-1 in myeloid progenitors is likely limited by inactivation of $E \mu$ due to lack of expression of critical activators necessary to maintain enhancer activity. It is interesting to note that Pim-1 driven by $v a v$ regulatory elements was low in mature myeloid cells [16].

Finally, although our flow cytometry analysis revealed that Pim1-deficiency does not provide a molecular explanation for the lymphoid/B cell deficiency in hoxa9-/- mice, pim1-/- and hoxa9-/- mice do share similar phenotypic and functional HSC defects. These observations and reported findings underscore developmental stage specific requirements for Hoxa9 and Pim-1 in hematopoietic development.

\section{Conclusions}

The goal of this study was to determine the requirement for Pim-1 in early lymphoid/B cell development. We show that controlled expression of Pim-1 is important in lymphoid/B cell development. High levels of Pim-1 are inhibitory while Pim-1 is dispensable for establishment of lymphoid progenitor and Pre-Pro-B cell pools.

\section{Abbreviations}

ALP, all lymphoid progenitors; BCP, B cell precursors; BLP, B lineage progenitors; $\mathrm{BM}$, bone marrow; CMP, common myeloid progenitor; flt3l, Flt3-ligand; GMP, granulocyte-macrophage progenitor; HSCs, hematopoietic stem cells; MEP, megakaryocyte-erythroid progenitor; MPP, multipotent progenitor; PPB, Pre-Pro-B; Tg, transgenic

\section{Acknowledgements}

Pim1-/- mice were provided by Andrew S. Kraft (Medical University of South Carolina) with permission obtained from Anton Berns (Netherlands Cancer Institute). EuPim-1 transgenic mice were provided by Jung-Hyun Park (Experimental Immunology Branch, $\mathrm{NCl}, \mathrm{NIH}$ ) with permission obtained from Anton Berns. This study was supported by R01 HL096108 to K.L.M.

Availability of data and materials

Not applicable.

\section{Authors' contributions}

$K L M$ conceived and designed the experiments, performed data analysis, made figures and wrote the manuscript. ZX performed experiments, data analysis and contributed to making the figures. KAG maintained the animal colonies and performed experiments. YL provided support for ZX. All authors read and approved the final manuscript.

\section{Competing interests}

The authors declare that they have no competing financial interests.

Consent for publication

Not applicable.

Ethics approval and consent to participate

All of the experiments using mice in this study were conducted under guidelines established and approved by the Mayo Clinic Institutional Animal Care and Use Committee.

\section{Author details}

${ }^{1}$ The Key Laboratory Pathobiology, Ministry of Education, Norman Bethune College of Medicine, Jilin University, Changchun 130000, People's Republic of China. ${ }^{2}$ Department of Immunology, College of Medicine, Mayo Clinic, 200 First Street SW, Rochester, MN 55905, USA. ${ }^{3}$ Department of Pathology, Norman Bethune College of Medicine, Jilin University, Changchun 130000, People's Republic of China.

Received: 14 January 2016 Accepted: 23 May 2016

Published online: 10 June 2016

\section{References}

1. Dolence JJ, Gwin K, Frank E, Medina KL. Threshold levels of Flt3-ligand are required for the generation and survival of lymphoid progenitors and B-cell precursors. Eur J Immunol. 2011;41(2):324-34.

2. Sitnicka E, Bryder D, Theilgaard-Monch K, Buza-Vidas N, Adolfsson J, Jacobsen SE. Key role of flt3 ligand in regulation of the common lymphoid progenitor but not in maintenance of the hematopoietic stem cell pool. Immunity. 2002;17(4):463-72.

3. So CW, Karsunky H, Passegue E, Cozzio A, Weissman IL, Cleary ML. MLL-GAS7 transforms multipotent hematopoietic progenitors and induces mixed lineage leukemias in mice. Cancer Cell. 2003;3(2):161-71.

4. Gwin KA, Shapiro MB, Dolence JJ, Huang ZL, Medina KL. Hoxa9 and Flt3 signaling synergistically regulate an early checkpoint in lymphopoiesis. J Immunol. 2013;191:745-54.

5. Cuypers HT, Selten G, Quint W, Zijlstra M, Maandag ER, Boelens W, van Wezenbeek P, Melief C, Berns A. Murine leukemia virus-induced T-cell lymphomagenesis: integration of proviruses in a distinct chromosomal region. Cell. 1984;37(1):141-50.

6. Eichmann A, Yuan L, Breant C, Alitalo K, Koskinen PJ. Developmental expression of pim kinases suggests functions also outside of the hematopoietic system. Oncogene. 2000;19(9):1215-24.

7. Mikkers $\mathrm{H}$, Nawijn M, Allen J, Brouwers $\mathrm{C}$, Verhoeven E, Jonkers J, Berns A. Mice deficient for all PIM kinases display reduced body size and impaired responses to hematopoietic growth factors. Mol Cell Biol. 2004;24(13):6104-15.

8. te Riele H, Maandag ER, Clarke A, Hooper M, Berns A. Consecutive inactivation of both alleles of the pim-1 proto-oncogene by homologous recombination in embryonic stem cells. Nature. 1990;348(6302):649-51.

9. Laird PW, van der Lugt NM, Clarke A, Domen J, Linders K, McWhir J, Berns A, Hooper M. In vivo analysis of Pim-1 deficiency. Nucleic Acids Res. 1993;21(20):4750-5. 
10. Domen J, van der Lugt NM, Acton D, Laird PW, Linders K, Berns A. Pim-1 levels determine the size of early B lymphoid compartments in bone marrow. J Exp Med. 1993;178(5):1665-73.

11. Dolence JJ, Gwin KA, Shapiro MB, Medina KL. Flt3 signaling regulates the proliferation, survival, and maintenance of multipotent hematopoietic progenitors that generate B cell precursors. Exp Hematol. 2014:42:380-93.

12. Li LX, Goetz CA, Katerndahl CD, Sakaguchi N, Farrar MA. A Flt3- and Rasdependent pathway primes B cell development by inducing a state of IL-7 responsiveness. J Immunol. 2010;184(4):1728-36.

13. Lilly M, Kraft A. Enforced expression of the Mr 33,000 Pim-1 kinase enhances factor-independent survival and inhibits apoptosis in murine myeloid cells. Cancer Res. 1997;57(23):5348-55.

14. Lilly M, Le T, Holland P, Hendrickson SL. Sustained expression of the pim-1 kinase is specifically induced in myeloid cells by cytokines whose receptors are structurally related. Oncogene. 1992;7(4):727-32.

15. Miura O, Miura Y, Nakamura N, Quelle FW, Witthuhn BA, Ihle JN, Aoki N. Induction of tyrosine phosphorylation of Vav and expression of Pim-1 correlates with Jak2-mediated growth signaling from the erythropoietin receptor. Blood. 1994;84(12):4135-41.

16. An N, Lin YW, Mahajan S, Kellner JN, Wang Y, Li Z, Kraft AS, Kang Y. Pim1 serine/threonine kinase regulates the number and functions of murine hematopoietic stem cells. Stem Cells. 2013;31(6):1202-12.

17. Lawrence HJ, Christensen J, Fong S, Hu YL, Weissman I, Sauvageau G, Humphries RK, Largman C. Loss of expression of the Hoxa-9 homeobox gene impairs the proliferation and repopulating ability of hematopoietic stem cells. Blood. 2005;106(12):3988-94.

18. Hu YL, Passegue E, Fong S, Largman C, Lawrence HJ. Evidence that the Pim1 kinase gene is a direct target of HOXA9. Blood. 2007;109(11):4732-8.

19. Linowes BA, Ligons DL, Nam AS, Hong C, Keller HR, Tai X, Luckey MA, Park $\mathrm{JH}$. Pim1 permits generation and survival of CD4+ T cells in the absence of gammac cytokine receptor signaling. Eur J Immunol. 2013;43(9):2283-94.

20. van Lohuizen M, Verbeek S, Krimpenfort P, Domen J, Saris C, Radaszkiewicz $T$, Berns A. Predisposition to lymphomagenesis in pim-1 transgenic mice: cooperation with c-myc and $\mathrm{N}$-myc in murine leukemia virus-induced tumors. Cell. 1989;56(4):673-82.

21. Gwin K, Dolence JJ, Shapiro MB, Medina KL. Differential requirement for Hoxa9 in the development and differentiation of $B, N K$, and DC-lineage cells from Flt3+ multipotential progenitors. BMC Immunol. 2013;14:5.

22. Kim KT, Baird K, Ahn JY, Meltzer P, Lilly M, Levis M, Small D. Pim-1 is upregulated by constitutively activated FLT3 and plays a role in FLT3-mediated cell survival. Blood. 2005;105(4):1759-67.

23. Medina KL, Tangen SN, Seaburg LM, Thapa P, Gwin KA, Shapiro VS. Separation of plasmacytoid dendritic cells from B-cell-biased lymphoid progenitor (BLP) and Pre-pro B cells using PDCA-1. PLOS ONE. 2013;8(10):e78408.

24. Medina KL, Garrett KP, Thompson LF, Rossi MI, Payne KJ, Kincade PW. Identification of very early lymphoid precursors in bone marrow and their regulation by estrogen. Nat Immunol. 2001;2(8):718-24.

25. Meeker TC, Nagarajan L, ar-Rushdi A, Rovera G, Huebner K, Croce CM. Characterization of the human PIM-1 gene: a putative proto-oncogene coding for a tissue specific member of the protein kinase family. Oncogene Res. 1987;1(1):87-101.

26. Bachmann M, Kosan C, Xing PX, Montenarh M, Hoffmann I, Moroy T. The oncogenic serine/threonine kinase Pim-1 directly phosphorylates and activates the G2/M specific phosphatase Cdc25C. Int J Biochem Cell Biol. 2006;38(3):430-43.

27. Amaravadi $\mathrm{R}$, Thompson CB. The survival kinases Akt and Pim as potential pharmacological targets. J Clin Invest. 2005;115(10):2618-24.

28. Matikainen S, Sareneva T, Ronni T, Lehtonen A, Koskinen PJ, Julkunen I. Interferon-alpha activates multiple STAT proteins and upregulates proliferation-associated IL-2Ralpha, c-myc, and pim-1 genes in human T cells. Blood. 1999;93(6):1980-91.

29. Block KM, Hanke NT, Maine EA, Baker AF. IL-6 stimulates STAT3 and Pim-1 kinase in pancreatic cancer cell lines. Pancreas. 2012:41(5):773-81.

30. Peltola KJ, Paukku K, Aho TL, Ruuska M, Silvennoinen O, Koskinen PJ. Pim-1 kinase inhibits STAT5-dependent transcription via its interactions with SOCS1 and SOCS3. Blood. 2004;103(10):3744-50

31. Iritani BM, Forbush KA, Farrar MA, Perlmutter RM. Control of $B$ cell development by Ras-mediated activation of Raf. Embo J. 1997;16(23):7019-31.

32. Morishita D, Katayama R, Sekimizu K, Tsuruo T, Fujita N. Pim kinases promote cell cycle progression by phosphorylating and down-regulating p27Kip1 at the transcriptional and posttranscriptional levels. Cancer Res. 2008;68(13):5076-85.
33. Lin YC, Jhunjhunwala S, Benner C, Heinz S, Welinder E, Mansson R, Sigvardsson M, Hagman J, Espinoza CA, Dutkowski J et al. A global network of transcription factors, involving E2A, EBF1 and Foxo1, that orchestrates B cell fate. Nat Immunol. 2010;11(7):635-43.

34. Mansson R, Welinder E, Ahsberg J, Lin YC, Benner C, Glass CK, Lucas JS, Sigvardsson M, Murre $C$ et al. Positive intergenic feedback circuitry, involving EBF1 and FOXO1, orchestrates B-cell fate. Proc Natl Acad Sci U S A. 2012;109(51):21028-33.

35. Wiesmann A, Phillips RL, Mojica M, Pierce $L$, Searles AE, Spangrude GJ, Lemischka I. Expression of CD27 on murine hematopoietic stem and progenitor cells. Immunity. 2000;12(2):193-9.

\section{Submit your next manuscript to BioMed Central and we will help you at every step:}

- We accept pre-submission inquiries

- Our selector tool helps you to find the most relevant journal

- We provide round the clock customer support

- Convenient online submission

- Thorough peer review

- Inclusion in PubMed and all major indexing services

- Maximum visibility for your research

Submit your manuscript at www.biomedcentral.com/submit
Biomed Central 